\title{
CONTROLLED PERIADVENTITIAL ADMINISTRATION OF VERAPAMIL INHIBITS NEOINTIMAL SMOOTH MUSCLE CELL PROLIFERATION AND AMELIORATES VASOMOTOR ABNORMALITIES IN EXPERIMENTAL VEIN BYPASS GRAFTS
}

Ron Brauner, $\mathrm{MD}^{\mathrm{a}}$

Hillel Laks, MD ${ }^{\mathrm{a}}$

Davis C. Drinkwater, Jr., MD ${ }^{\mathrm{a}}$

Gautam Chaudhuri, MD ${ }^{\mathrm{a}}$

Oleg Shvarts, MS $^{\mathrm{a}}$

Thomas Drake, $\mathrm{MD}^{\mathrm{a}}$

Sunita Bhuta, MD ${ }^{\mathrm{a}}$

David Mishaly, MD $^{\mathrm{b}}$

Ilia Fishbein, $\mathrm{MD}^{\mathrm{b}}$

Gershon Golomb, $\mathrm{PhD}^{\mathrm{b}}$
Objective: Inhibition of early myointimal proliferation may improve longterm patency of vein grafts, but the clinical use of many experimental drugs is limited by systemic toxicity. To determine whether this goal can be achieved by low-dose targeted drug administration, we constructed a polymeric system delivering verapamil and evaluated the effects on local and downstream vein graft morphology, neointimal smooth muscle cell proliferation, and vasomotor function. Methods: Ethylene-vinyl acetate polymeric delivery systems were constructed, containing $2 \%$ verapamil by weight. These are flexible, biocompatible, and nonbiodegradable matrices, delivering the drug at a rate of $10 \mu \mathrm{g} /$ day. The autologous external jugular vein was used to create a carotid artery bypass graft in hypercholesterolemic $(n=22)$ rabbits. Verapamil-containing matrices $(n=12)$ or plain polymers (control, $n=10$ ) were wrapped around the proximal third of the veins after reperfusion. Graft vasomotor function was evaluated and was also compared with function of an additional group of normocholesterolemic vein grafts $(n=8)$. Results: Twenty-eight days after grafting, intimal index (intima/media thickness ratio) was $31 \%$ lower, neointima/original lumen surface ratio was $26 \%$ lower, and residual luminal area was $71 \%$ greater $\left(4.00 \pm 1.2 \mathrm{~mm}^{2}\right.$ versus $2.34 \pm 0.9 \mathrm{~mm}^{2}$, all $\left.p<0.01\right)$ under verapamil matrices compared with control grafts. Neointimal smooth muscle cell content was reduced from $45.4 \%$ to $28.2 \%$, and net neointimal smooth muscle cell thickness was reduced by $47 \%(30 \mu \mathrm{m}$ vs $15.8 \mu \mathrm{m}$, both $p<0.01$. Verapamil-treated segments distal to the matrices also showed significantly lower neointimal smooth muscle cell density and increased lumen size. Sensitivity to serotonin and vasomotor responses to serotonin, norepinephrine, and sodium nitroprusside in distal segments were significantly lower in verapamil-treated grafts than in controls. Conclusions: Periadventitial controlled administration of verapamil below $1 \%$ of the systemic dose effectively inhibits myointimal hyperplasia in vein grafts. Local polymeric drug delivery may be readily applicable to coronary revascularization operations. (J Thorac Cardiovasc Surg 1997;114:53-63)
From the Division of Cardiothoracic Surgery, University of California at Los Angeles School of Medicine, Los Angeles, Calif., ${ }^{a}$ and the School of Pharmacy, the Hebrew University of Jerusalem, Jerusalem, Israel. ${ }^{\mathrm{b}}$

Presented at the Sixty-ninth Scientific Sessions of the American Heart Association, New Orleans, La., November 1996.

Received for publication Dec. 23, 1996; revisions requested Jan. 23, 1997; revisions received Feb. 13, 1997; accepted for publication Feb. 21, 1997.

Address for reprints: Hillel Laks, MD, Division of Cardiothoracic Surgery, UCLA Medical Center, 62-182A Center for the Health Sciences, 10833 Le Conte Ave., Los Angeles, CA 90095.

Copyright $(\mathcal{O} 1997$ by Mosby-Year Book, Inc.

0022-5223/97 $\$ 5.00+0 \quad \mathbf{1 2 / 1 / 8 1 3 9 0}$
$\Lambda$ utologous saphenous veins remain the most Awidely used conduits for coronary revascularization, but their long-term patency is limited by the development of atherosclerosis. This leads to occlusion of up to $50 \%$ of vein grafts within 10 years and to atherosclerotic disease in $13 \%$ of patent grafts at 3 years, $27 \%$ at 8 years, and $50 \%$ at 12 years. ${ }^{1}$ Early morphologic changes in arterialized veins include the development of intimal hyperplasia, which may constitute the substrate for subsequent development of accelerated atherosclerosis. ${ }^{2,3}$ Although this is initially a remodeling process precipitated by the altered hemodynamic conditions in the arterial cir- 
culation and by resulting endothelial damage, intimal hyperplasia may also result in significant luminal narrowing and graft failure. Interference with the early proliferative phase of neointima formation may delay the occurrence of vein graft atherosclerosis and improve long-term patency.

Many systemically administered pharmacologic agents have been shown to inhibit intimal hyperplasia in experimental vein grafts. However, some of the more potent drugs (e.g., antimetabolites) have severe systemic side effects at the dose required to achieve an efficient vascular effect, whereas others are not readily absorbed after oral intake or have a short plasma half-life. ${ }^{4} \mathrm{~A}$ targeted drug delivery system acting on the grafted conduit may circumvent these limitations by achieving high drug concentrations locally. Such systems typically deliver $1 \%$ or less of the systemic drug dose, minimizing side effects and increasing cost-effectiveness. ${ }^{5}$ In coronary surgical revascularization, the application of a local drug release system at the time of the operation seems particularly attractive: in addition to the benefits of targeted drug delivery, a nonabsorbable polymer may protect the conduit from accidental injury and facilitate dissection in the event of resternotomy. Rigid external support may confer an additional local inhibitory effect on intimal hyperplasia. ${ }^{6}$ A distal downstream effect is also desirable, because it would enable application of the device to proximal segments of the conduit only, avoiding the need for matrix modeling. Finally, this strategy does not require patient compliance and may therefore benefit older or disabled patients who require coronary artery bypass grafting.

Ethylene-vinyl acetate (EVA)-based periadventitial matrices delivering heparin ${ }^{7}$ and more recently AG-17, an inhibitor of protein tyrosine kinase activity, ${ }^{8}$ were shown to inhibit neointimal formation in the balloon carotid arterial injury model in rats. Other polymeric extraluminal delivery systems have been successfully used in arterial injury models when systemic administration was ineffective., 10 Verapamil, a widely used calcium-channel blocker, was previously shown to inhibit intimal hyperplasia in normocholesterolemic rabbit arterialized vein grafts. ${ }^{11}$ Although their precise mechanism of action is unknown, this and other calcium-channel blockers have been reported to interfere with vascular smooth muscle cell (SMC) proliferation and migration induced by platelet-derived growth factor (PDGF) in vitro. ${ }^{12}$ Moreover, a decrease in cellular lipid content was attributed to verapamil stimulation of cholesteryl ester hydrolase activity in SMCs, and inhibition of vascular SMC proliferation and migration was observed in rabbits. ${ }^{13,14}$

In the present study we constructed a periadventitial polymeric system delivering verapamil and evaluated its ability to affect early vein graft myointimal proliferation, lipid accumulation, and vasomotor function in hypercholesterolemic rabbits. We also sought to determine whether an effect would be limited to the graft segment directly under the polymer or whether it could also be detected downstream.

\section{Materials and methods}

Polymeric drug delivery system. EVA copolymer (Elvax 40, DuPont Pharmaceuticals, Wilmington, Del.) was washed with water and alcohol to remove impurities. ${ }^{15}$ Verapamil hydrochloride (Sigma Chemical Co., St. Louis, Mo.) and EVA were dissolved in methylene chloride, yielding a $15 \% \mathrm{wt} / \mathrm{vol}$ solution as described previously. 8,15 The solution was poured into glass Petri dishes and dried. The cast polymeric matrix $(0.2 \mathrm{~mm}$ thick) was cut into circular pieces $\left(0.79 \mathrm{~cm}^{2}\right)$ for in vitro release studies and into rectangular pieces $(2 \times 0.7 \mathrm{~cm}$ and weighing $100 \mathrm{mg})$ for perivascular implantation. Unidirectional release of the drug was achieved by sealing one surface of the matrices with blank EVA film using a few drops of methylene chloride as glue. The resulting delivery system is a flexible transparent matrix having the advantages of being biocompatible and nondegradable. ${ }^{15}$ Before implantation the matrices were sterilized with ethylene oxide.

Drug release kinetics. Release kinetics were measured in this fashion: Two circular pieces of sealed verapamil matrices were separated by aluminum foil and mounted back to back between two halves of a diffusion cell as described previously. ${ }^{8}$ The cells were filled with HEPES buffer, $\mathrm{pH} 7.4$, placed in a shaker at $37^{\circ} \mathrm{C}$, and the amount released from each matrix over time was measured in the receiving solution by spectrophotometry at $233 \mathrm{~nm}$. To keep sink conditions (maximum concentration of $10 \%$ of saturation), we replaced the receiving liquid at each time point with fresh buffer.

Animal model. Thirty New Zealand White rabbits weighing 4 to $4.5 \mathrm{~kg}$ (Irish Farms, Norco, Calif.) were used to create experimental vein bypass grafts of the common carotid artery. Anesthesia was induced intravenously with ketamine $(10 \mathrm{mg} / \mathrm{kg})$ and acepromazine $(1 \mathrm{mg} / \mathrm{kg})$ and maintained with inhaled $1.5 \%$ isoflurane in oxygen by means of mechanical ventilation. Heparin $(200 \mathrm{mg} / \mathrm{kg}$ ) and cefazolin $(40 \mathrm{mg} / \mathrm{kg})$ were administered intravenously before incision. Exposure and dissection of the left external jugular vein and common carotid artery were completed by way of a paramedian neck incision. A segment of the vein extending cephalad from the junction of the external and internal jugular veins $(2.5 \mathrm{~cm}$ long) was excised and preserved in lactated Ringer's solution with papaverine $(0.8 \mathrm{mg} / \mathrm{ml})$. Proximal and distal anastomoses were performed in an end-to-side fashion with 8-0 
polypropylene sutures (Deknatel Inc., Division of DSP Worldwide, Fall River, Mass.). The bypassed carotid artery segment was excised and graft patency was confirmed by ultrasonic flow measurement (Transonic Systems, Inc., Ithaca, N.Y.). Hypercholesterolemic rabbits $(n=22)$ received a $1 \%$ cholesterol-supplemented diet (Newco, Fontana, Calif.), which was started 28 days before the operation and continued throughout the study. These animals were randomized to receive polymeric EVA matrices containing verapamil $(n=12)$ or control, plain polymers $(n=10)$. After completion of the anastomoses and restoration of flow, the polymer patches were applied around the proximal third of the grafts and the free edge was approximated with a 6-0 polypropylene suture, care being taken not to compress or deform the vein (Fig. 1). The matrices were anchored to the proximal carotid artery stump to prevent distal migration of the polymer. An additional group of normocholesterolemic rabbits $(n=8)$ had similar procedures but no polymer implantation. The skin was closed with 3-0 Dexon suture (Davis \& Geck, Danbury, Conn.). All animals were treated in compliance with the "Principles of Laboratory Animal Care" formulated by the National Society for Medical Research and the "Guide for the Care and Use of Laboratory Animals" prepared by the Institute of Laboratory Animal Resources and published by the National Institutes of Health (NIH Publication No. 86-23, revised 1985).

Specimen processing. Twenty-eight days after the operation, animals were put to death and graft tissue was collected for morphometric and vasomotor analysis. Vein graft regions within $3 \mathrm{~mm}$ of the proximal or distal suture lines were discarded. Specimens were divided into the experimental groups as follows: $\mathrm{HC}_{\text {control }}$-hypercholesterolemic rabbits, proximal graft segments directly under control EVA polymers; $\mathrm{HCV}_{\text {proximal }}$-hypercholesterolemic rabbits, proximal graft segments directly under verapamil matrices; and $\mathrm{HCV}_{\text {distal }}$ hypercholesterolemic rabbits, graft segments at least $4 \mathrm{~mm}$ distal to the verapamil matrices. Normocholesterolemic (NC) vein graft midsegments were used for vasomotor studies only. Tissue processing personnel were blinded to the experimental protocol.

For light microscopy, graft rings from under the polymer and from distal area midsegments were fixed in $10 \%$ formalin for at least 24 hours and then embedded in paraffin. Ten-micron sections were cut perpendicular to the vessel wall and then stained with Masson's trichrome stain. Four to eight separate sections of each segment (under and distal to the polymers) at $200 \mu \mathrm{m}$ intervals were analyzed and average values were derived for each specimen. Morphometric measurements of average intimal and medial thickness and areas, as well as lumen contour length, were obtained by means of computed image analysis software (Timwin, TEA, Dordrecht, The Netherlands). Residual luminal area was calculated from the lumen contour. The degree of neointimal thickening was expressed as (1) the ratio of the neointimal area and the original luminal area as defined by the outer neointimal margin (percent stenosis) and (2) the ratio of the neointimal and medial surfaces (intimal index).
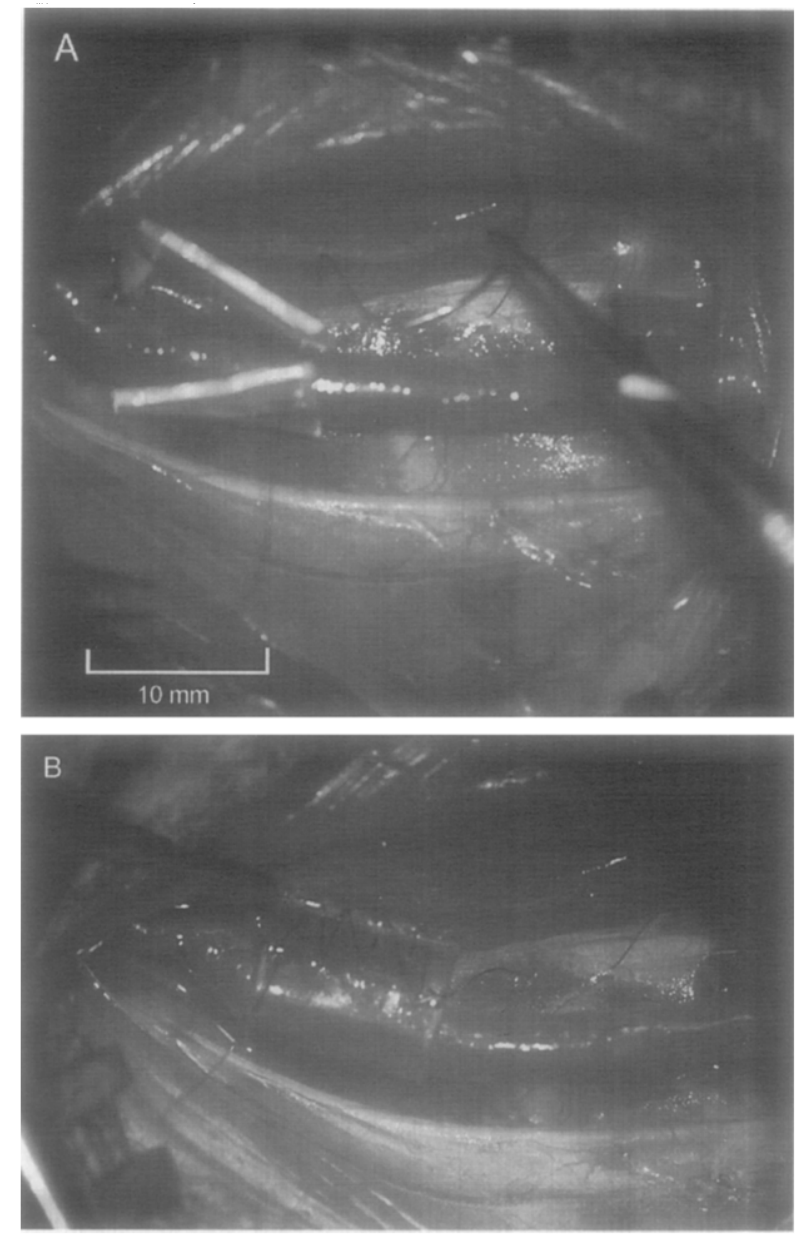

Fig. 1. A, Placement of a verapamil-EVA polymeric matrix around the proximal third of a jugular vein graft bypassing the carotid artery. The matrix is flexible and provides good suture handling. B, Final appearance of the graft with the matrix in place. Anchoring the polymer to the proximal carotid stump (not seen) prevents distal migration. At reoperation, dissection was facilitated by the presence of the device.

For transmission electron microscopy, graft rings were immersed in $2.5 \%$ glutaraldehyde and $2 \%$ paraformaldehyde buffered by sodium cacodylate. Tissue was then postfixed in $1 \%$ osmium, dehydrated, and embedded in an epoxy resin. One-micron sections stained with toluidine blue were initially reviewed in a blinded fashion to identify intimal areas suitable for ultrastructural study. Ultrathin sections were stained with uranyl acetate and lead citrate. Electron micrographs were prepared on a Zeiss EM 109 electron microscope (Carl Zeiss Inc., Thornwood, N.Y.) and printed at a final magnification of $\times 3000$. Twelve to sixteen random nonadjacent captures within intimal areas were analyzed for each specimen. SMCs were identified by the characteristic appearance of actin filaments with mostly peripheral local densities, by the presence of a discontinuous external lamina and dense rough endoplas- 


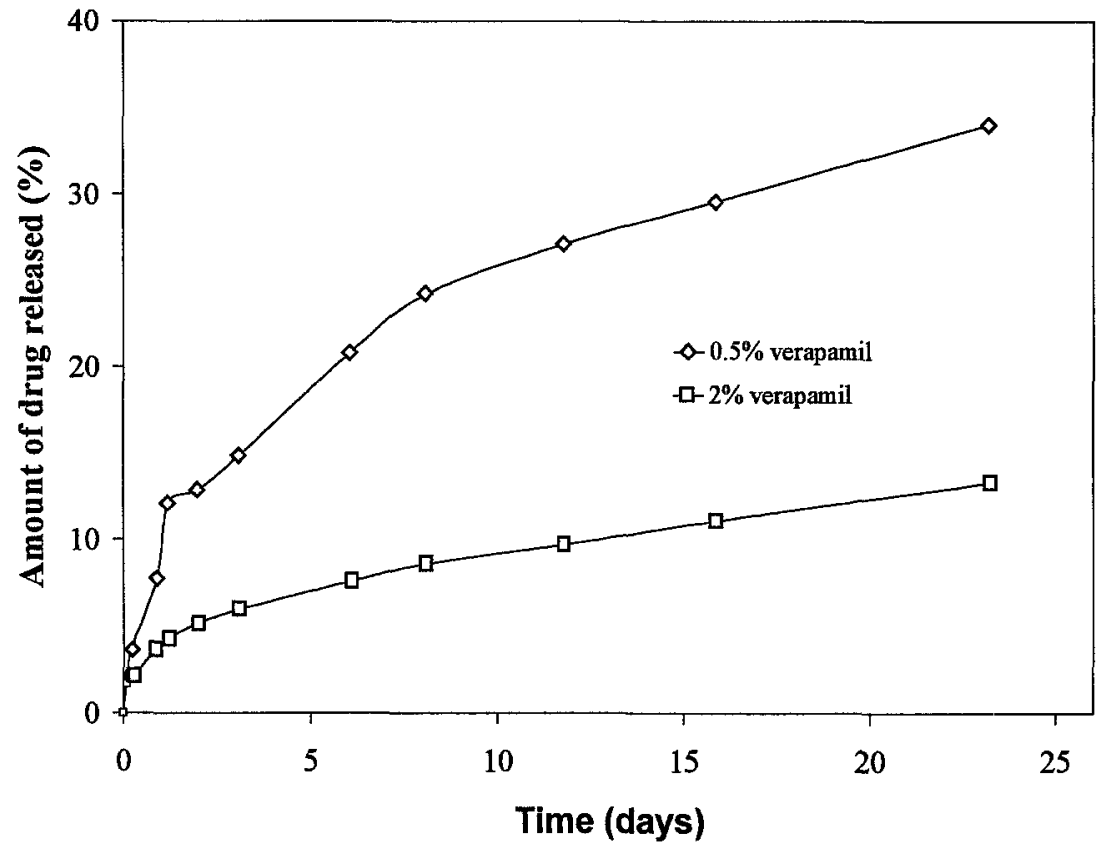

Fig. 2. Release kinetics of EVA polymeric systems $(0.2 \mathrm{~mm}$ thick) containing $0.5 \%$ or $2 \% \mathrm{wt} / \mathrm{wt}$ of verapamil. Two percent verapamil matrices were used in the present study. Drug release rate decreases over time, with an initially high delivery rate that may be beneficial at the early stages of vascular injury.

mic reticulum, and by the absence of lysosomes. To determine intimal SMC content, we subjected the photographs to computer-assisted morphometric examination (Timwin, TEA) and the fractional area occupied by SMCs (SMC density) was determined. An estimate of the absolute mass of SMCs and matrix in the graft intima was thus obtained.

Ex vivo isometric vasomotor studies. Vein graft segments measuring 4 to $5 \mathrm{~mm}$ were collected from the distal portion of the grafts, ending at least $3 \mathrm{~mm}$ proximal to the distal anastomosis site, and were immediately placed in oxygenated Krebs solution (millimolar concentrations: $\mathrm{NaCl} 118 ; \mathrm{KCl} 4.7 ; \mathrm{MgSO}_{4} 1.2 ; \mathrm{CaCl}_{2} 2.5 ; \mathrm{NaHCO}_{3} 25 ;$ $\mathrm{KH}_{2} \mathrm{PO}_{4} 1.2$, glucose 11.5 ). The solution was continuously oxygenated with a $95 \%$ oxygen and $5 \%$ carbon dioxide gas mixture and maintained at $37^{\circ} \mathrm{C}$ throughout the experiment. The rings were suspended on two stainless steel hooks in $25 \mathrm{ml}$ tissue baths (Radnoti Glass Technologies, Monrovia, Calif.) with one hook fixed to the bottom of the bath and the other connected to a multichannel polygraph recorder (Grass Instruments Inc., Quincy, Mass.), then set at a tension of $1 \mathrm{gm}$ and allowed to equilibrate for 1 hour, while the Krebs solution was replaced every 20 minutes. After equilibration, the optimal resting tension for maximal response was determined by recording the contractile response to a Krebs solution with a $\mathrm{KCl}$ concentration of $60 \mathrm{mmol} / \mathrm{L}$ at different resting tensions, ranging from 0.5 to $2.5 \mathrm{gm}$ in $0.5 \mathrm{gm}$ increments. The tension at which a ring generated the maximal contraction was considered to be the optimal resting tension for that ring, and the ring was set at that tension for subsequent studies. After washouts and equilibration, dose-response curves were obtained for cumulative doses of serotonin $\left(10^{-8}\right.$ to $10^{-4}$ $\mathrm{mol} / \mathrm{L})$ and norepinephrine $\left(10^{-8}\right.$ to $\left.10^{-4} \mathrm{~mol} / \mathrm{L}\right)$. After the grafts had been precontracted to $80 \%$ of the maximal tension generated to norepinephrine, the relaxation response to sodium nitroprusside $\left(10^{-12}\right.$ to $\left.10^{-8} \mathrm{~mol} / \mathrm{L}\right)$ was determined. All chemicals were purchased from Sigma Chemical Co., St. Louis, Missouri, and were dissolved in distilled water.

Data analysis. Isometric responses of the vein rings (in milligrams of generated force) were adjusted to the maximal ring contraction to a Krebs buffer solution with a $60 \mathrm{mmol} / \mathrm{L}$ concentration of $\mathrm{KCl}$ and expressed as percentage of the maximal response. The agonist concentration at which half-maximal contraction or relaxation occurred $\left(E_{50}\right)$ was calculated from the response curves by logistic analysis. Graft sensitivity to each agonist was defined as $-\log _{10}\left(\mathrm{EC}_{50}\right)$. Data are expressed as mean \pm standard deviation of the mean. Comparisons of morphometric data from local and distal verapamil-treated segments and from control segments were made by one-way analysis of variance, and significant differences were analyzed with Dunnett's post hoc test for multiple comparisons. Sensitivity and contraction responses in verapamiltreated vein grafts were compared with control responses by means of the two-tailed Student's $t$ test. Significance level was set at $p<0.05$.

\section{Results}

Verapamil delivery system. EVA matrices containing verapamil were homogeneous, and examination of their structure by scanning electron micros- 
copy did not detect any drug crystals or particles, which suggests that an amorphous dispersion of verapamil in the polymer is produced when the agent and EVA are cast from methylene chloride solution. The drug release kinetics and the effect of drug load concentration on the release rate kinetics were determined to enable a rational design of a drug delivery system responding to the therapeutic results in vivo. Matrices loaded with $0.5 \%$ and $2 \%$ $\mathrm{wt} / \mathrm{wt}$ released the drug in an exponential manner, that is, release rate decreased over time (Fig. 2). As seen, the fraction released over time is affected by the drug load: after 24 days, $13 \%$ and $34 \%$ of the drug load was released from $2 \%$ and $0.5 \%$ matrices, respectively. On the basis of these results, matrices containing $2 \%$ verapamil and releasing approximately $10 \mu \mathrm{g} /$ day were used for in vivo implantation studies.

Reoperation. The cholesterol-supplemented diet was well tolerated by all animals. Serum cholesterol levels at time of the initial operation and before the animals were put to death were $1460 \pm 384 \mathrm{mg} / \mathrm{dl}$. At reoperation, all grafts were found to be patent and no distal migration of the EVA polymers was noted. Adhesions formed around the grafts in all animals; however, hypercholesterolemic rabbits were noted to have denser adhesions and more lipid deposition in subcutaneous tissues. Localization, exposure, and dissection of the grafts were greatly facilitated by the presence of EVA polymers. Whereas dense adhesions formed around the unwrapped segments of all grafts, connective tissue around the EVA matrices did not adhere to the biocompatible polymers, and these could be easily mobilized. No inflammatory response or granulation tissue developed. In the wrapped region of the grafts, the presence of EVA matrices virtually eliminated the danger of inadvertent injury to the vessel during reoperation. The matrices did not constrict the grafts, and adhesions did not form between the graft adventitia and the inner polymer surface. After removal of the polymers, the demarcation line between the area beneath and distal to the matrices could be seen and was used to determine the respective areas for specimen collection.

Morphology. The neointima consisted of proliferated SMCs ("myointimal cells"), lipid-laden phagocytic cells, and a loose extracellular matrix. Representative photomicrographs of $\mathrm{HC}_{\text {control, }}$ $\mathrm{HCV}_{\text {proximal }}$, and $\mathrm{HCV}_{\text {distal }}$ segments are shown in Fig. 3, and wall cross sections are shown in Fig. 4. The effects of verapamil treatment on vein graft

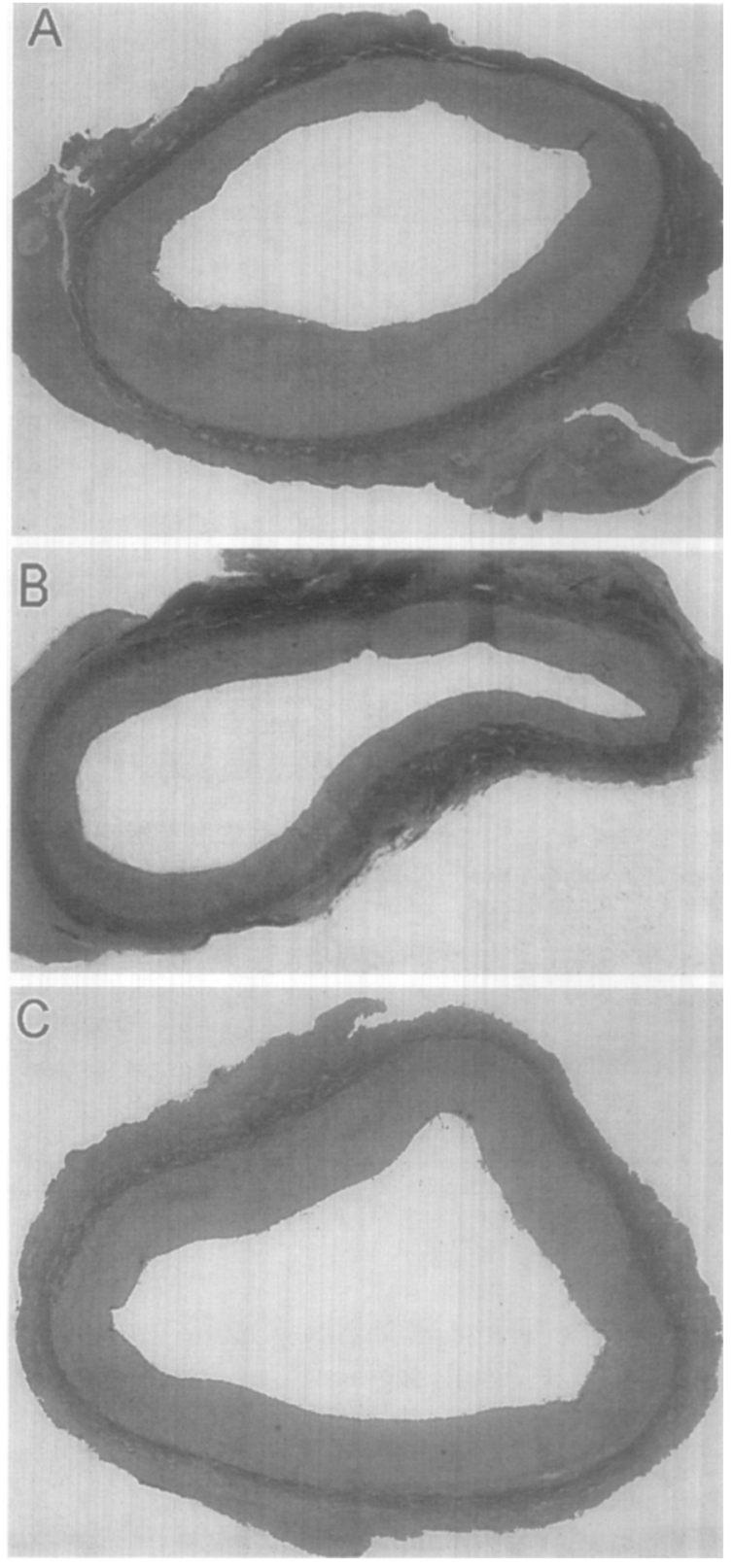

Fig. 3. Representative photomicrographs of vein graft segments directly under plain control EVA polymers (A), under verapamil-EVA matrices (B), and distal segments of verapamil-treated grafts $(\mathbf{C})$.

remodeling 28 days after the operation are shown in Table I. The intimal index and percent stenosis under the polymer decreased significantly, reflecting an inhibitory effect on intimal thickening. A $20 \%$ decrease in average intimal width (314 $\pm 68 \mu \mathrm{m}$ vs $392 \pm 122 \mu \mathrm{m}$ ) was also observed but was not statistically significant. These effects were not observed distal to the polymers. However, residual 

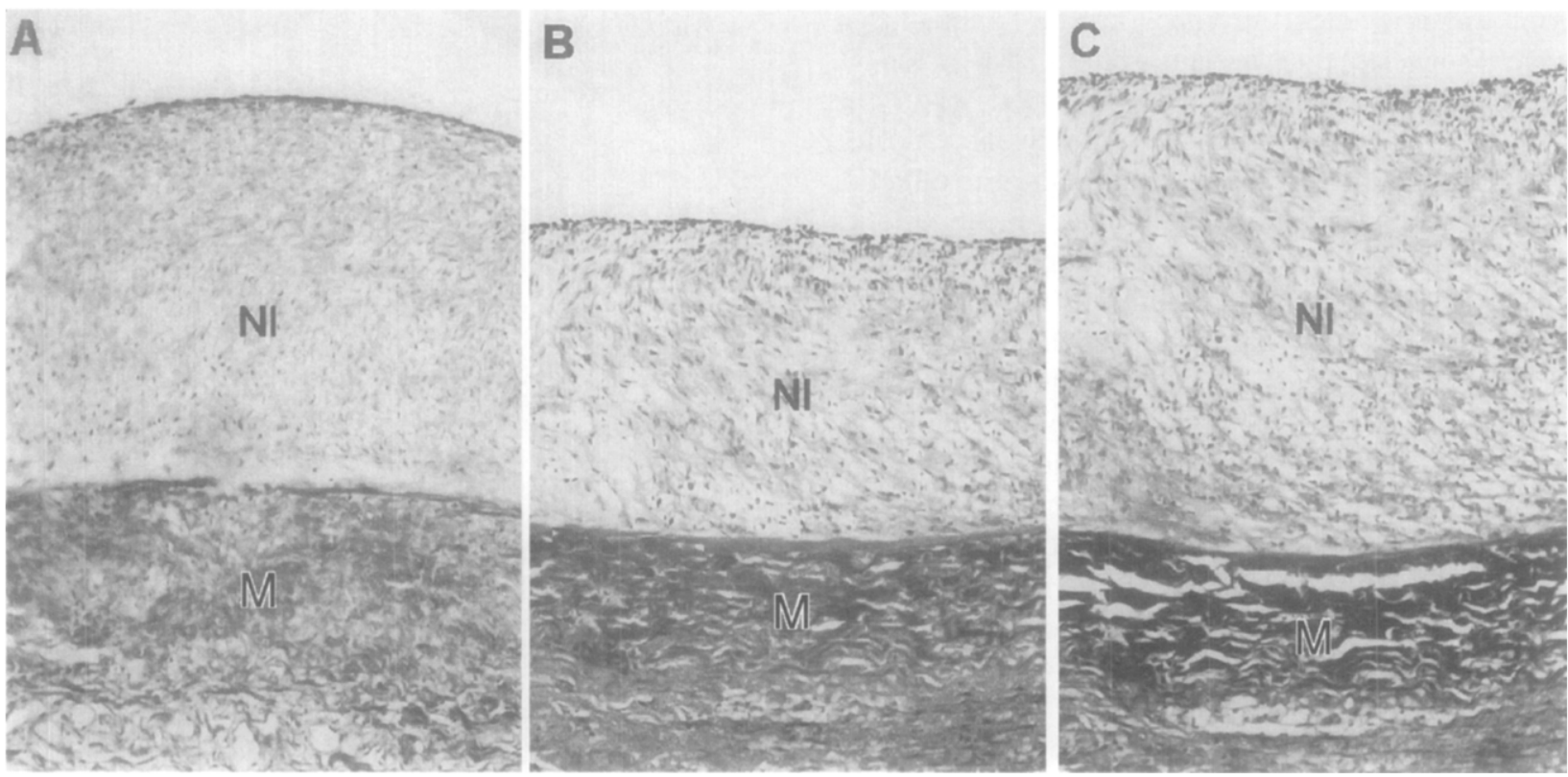

Fig. 4. Cross sections of the vein graft wall under control EVA (A), under verapamil-EVA (B), and downstream of verapamil-EVA polymeric matrices (C). NI, Neointima; $M$, media.

Table I. Morphometric analysis of proximal and downstream verapamil-treated and control vein graft segments

\begin{tabular}{llll}
\hline & $H C_{\text {control }}$ & $H C V_{\text {proximal }}$ & $H C V_{\text {dissal }}$ \\
\hline Intimal index & $2.83 \pm 0.49$ & $1.94 \pm 0.46^{*}$ & $2.63 \pm 0.61$ \\
Percent stenosis & $50.0 \pm 8$ & $37.1 \pm 7^{*}$ & $50.4 \pm 6$ \\
Luminal area $\left(\mathrm{mm}^{2}\right)$ & $2.34 \pm 0.92$ & $4.00 \pm 1.25^{*}$ & $3.37 \pm 0.51^{*}$ \\
\hline
\end{tabular}

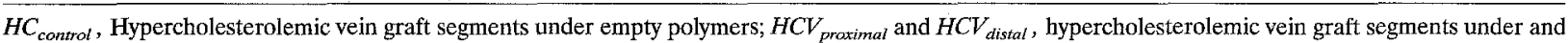
distal to verapamil-EVA polymers. Values are the mean \pm standard deviation.

${ }^{*} p<0.01$ compared with $H C_{\text {control }}$ grafts.

luminal area was significantly greater in both the wrapped and distal segments in the verapamiltreated group.

Representative electron micrographs of intimal cross sections from $\mathrm{HC}_{\text {control, }} \mathrm{HCV}_{\text {proximal }}$, and $\mathrm{HCV}_{\text {distal }}$ vein grafts are shown in Fig. 5. Neointimal SMC density (percent SMC surface from total area) was significantly lower in verapamil-treated grafts, an effect observed both under the polymers and downstream. When excluding extracellular matrix and foam cells, the amount of net intimal SMC (defined as SMC density $\times$ mean intimal thickness) was also significantly lower under the verapamil matrices. Despite the lower SMC density distal to the EVA polymers, the net mass of neointimal SMC was not significantly lower in this area of the graft. This was due to the greater average intimal thickness resulting from significantly higher lipid and foam cell accumulation in the distal segments (Table II).
Vasomotor function. Isometric tension studies were performed on vein graft segments distal to the polymeric matrices, and an additional group of normocholesterolemic vein grafts were used for comparison. As can be seen in Table III, hypercholesterolemia significantly increased vein graft sensitivity to serotonin and decreased graft sensitivity to sodium nitroprusside. A lower sensitivity to norepinephrine was also observed but the difference was not statistically significant. The effects of hypercholesterolemia on serotonin and norepinephrine sensitivity were reversed by verapamil treatment to levels resembling those of normocholesterolemic vein grafts. The lower sensitivity to sodium nitroprusside was not significantly affected by verapamil.

The absolute contraction force (milligrams) generated to increasing concentrations of serotonin and norepinephrine and relaxation to sodium nitroprusside were significantly higher in hypercholesterolemic controls than in normocholesterolemic ani- 
mals. This enhanced contractile response was reversed in verapamil-treated grafts, with the observed reduction in generated force being significant for all studied agonists (Table IV). The maximal response to a high $\mathrm{KCl}(60 \mathrm{mmol} / \mathrm{L})$ solution was highly variable; therefore the higher contraction observed in hypercholesterolemic grafts was not statistically significant. Contraction responses were then adjusted to the maximal $\mathrm{KCl}$-induced contraction (Table V). Adjusted contraction to serotonin was significantly enhanced by hypercholesterolemia, and verapamil treatment resulted in significantly reduced contraction force to serotonin, at levels similar to those of normocholesterolemic controls (Fig. 6). Both KCl-adjusted contraction to norepinephrine and relaxation of precontracted grafts to sodium nitroprusside were similar in control and verapamil-treated grafts.

\section{Discussion}

The present study demonstrates the ability of a polymeric targeted drug delivery system implanted at surgery to inhibit intimal proliferation in experimental vein grafts. Local effects included a significant reduction in neointimal SMC mass and density and a more favorable graft remodeling, expressed by reduced intima/media ratio and narrowing of the original lumen (percent stenosis). A distal downstream effect was also noted, consisting mainly of improvement in some of the vasomotor abnormalities induced by vein arterialization and hypercholesterolemia. This effect was achieved with an average drug release rate of $10 \mu \mathrm{g} / \mathrm{day}$, less than $1 \%$ of the intravenous dose used by El-Sanadiki and associates ${ }^{11}$ to achieve a similar effect. In addition to being both safe and therapeutic, the system facilitated vessel localization and dissection at reoperation.

After implantation in the arterial circulation, both human and experimental vein grafts undergo a type II vascular injury, characterized by endothelial and intimal damage. Patchy endothelial denuding seen immediately after implantation results in loss of the critical inhibitory effect of this layer on SMC growth. ${ }^{16}$ As early as 1 hour after the operation, adherence of blood-borne cells, particularly platelets and monocytes, initiates an inflammatory cascade within the vessel wall. ${ }^{2}$ Under the influence of locally secreted growth factors such as PDGF and transforming growth factor $-\beta$, medial SMCs undergo a phenotypic change from the contractile to a synthetic phenotype, resulting in migration and intimal proliferation of the modified "myointimal"
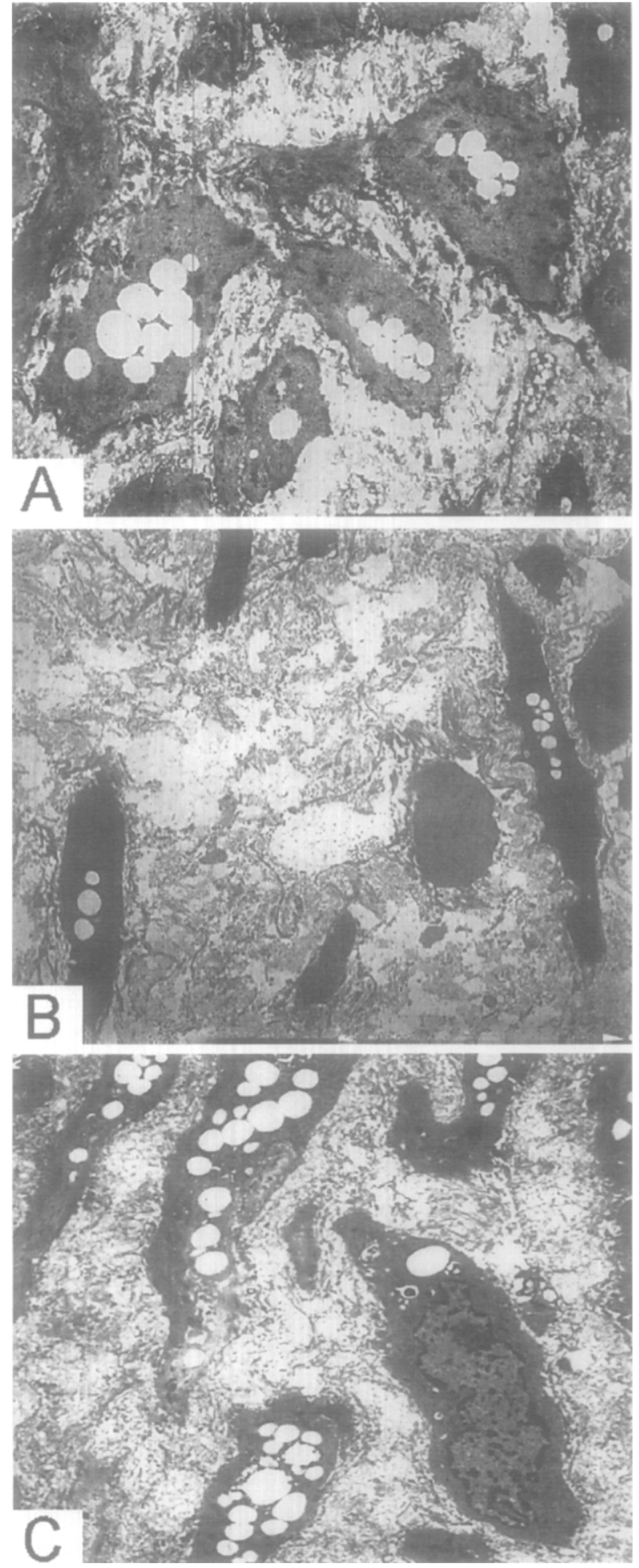

Fig. 5. Representative transmission electron micrographs of vein graft intima. All visualized cells are proliferated smooth muscle cells. A, Directly under empty control EVA matrices. B, Under verapamil-EVA matrices. C, Distal segments of verapamil-treated grafts. 
Table II. Neointimal SMC content in proximal and downstream verapamil-treated and control vein graft segments

\begin{tabular}{lccc} 
& $H C_{\text {control }}$ & $H C V_{\text {proximal }}$ & $H C V_{\text {distal }}$ \\
\hline Intimal SMC density $(\%)$ & $45.4 \pm 6.7$ & $28.2 \pm 6.4^{*}$ & $33.0 \pm 7.3^{*}$ \\
Net intimal SMC $(\mu \mathrm{m})$ & $174 \pm 49$ & $88 \pm 25^{*}$ & $1.53 \pm 59$ \\
Matrix and foam cells $(\mu \mathrm{m})$ & $216 \pm 81$ & $229 \pm 57$ & $303 \pm 59 \dagger$
\end{tabular}

$S M C$, Smooth muscle cells. Values are the mean \pm standard deviation. ${ }^{*} p<0.01$ compared with $H C_{\text {control }}$ segments.

$\neq p<0.05$ compared with $H C_{\text {control }}$ and $p=0.01$ compared with $H C V_{\text {proximal }}$ segments.

Table III. Sensitivity of normocholesterolemic, hypercholesterolemic control, and verapamil-treated distal vein graft segments

\begin{tabular}{lccc}
\hline & $N C$ & $H C_{\text {contral }}$ & \multicolumn{1}{c}{$H C V_{\text {distat }}$} \\
\hline Serotonin & $5.39 \pm 0.28$ & $6.35 \pm 0.29$ & $5.80 \pm 0.38^{*}$ \\
Norepinephrine & $5.60 \pm 0.79$ & $5.01 \pm 0.71$ & $5.94 \pm 0.85 \dagger$ \\
Sodium nitroprusside & $11.68 \pm 0.54 \ddagger$ & $10.62 \pm 0.43$ & $10.74 \pm 0.45$
\end{tabular}

The sensitivity of normocholesterolemic $(N C)$, hypercholesterolemic controls $\left(H C_{\text {control }}\right)$, and verapamil-treated $\left(H C V_{\text {discal }}\right)$ vein grafts to various agonists is expressed as $-\log _{10}\left(E_{50}\right)$. Values represent the mean \pm standard deviation.

${ }^{*} p=0.002$ and $\dagger p=0.04$ compared with $H C_{\text {control }}$ segments. $\ddagger p<0.05$ compared with both hypercholesterolemic segments.

cells. ${ }^{16}$ In saphenous vein grafts, the early fibrointimal hyperplastic process directly results in significant graft narrowing (a $25 \%$ reduction in luminal diameter at 1 year is common) and is considered responsible for the subacute graft occlusions seen in $10 \%$ of grafts 1 to 12 months after the operation. ${ }^{3}$ However, the proliferative phase is short-lived, peaking at 2 to 21 days in rodents and 1 to 12 months in human beings, after which return to a quiescent chronic phase is seen. In the rabbit, no further increase in intimal cell mass and DNA content occurs after 4 weeks, and subsequent increase in wall mass and late remodeling are due to connective tissue accumulation and deposition of lipid, for which vein grafts seem to have a high affinity. ${ }^{2,3,17}$ On this basis it has been assumed that successful interference with the early proliferative burst may have a beneficial effect on intermediate and late graft patency, but the latter remains currently unproved. Documentation of late progression of intimal hyperplasia to atherosclerosis in mature grafts is largely based on indirect angiographic follow-up, and it is not known whether sites of foam cell deposition are the specific precursor lesions to the fully developed complex atherosclerotic plaque. ${ }^{3}$

Although hypercholesterolemia does not seem to
Table IV. Maximal absolute contraction/relaxation force $(\mathrm{mg})$ of normocholesterolemic, hypercholesterolemic control, and verapamil-treated vein graft segments

\begin{tabular}{lcrc}
\hline & $N C$ & $H C_{\text {controt }}$ & \multicolumn{1}{c}{$H C V_{\text {distal }}$} \\
\hline $\mathrm{KCl} 60 \mathrm{mmol} / \mathrm{L}$ & $289 \pm 104$ & $510 \pm 311$ & $380 \pm 340$ \\
Serotonin & $381 \pm 206$ & $2111 \pm 400^{*}$ & $1369 \pm 666 \S$ \\
Norepinephrine & $253 \pm 157$ & $531 \pm 296 \dagger$ & $298 \pm 199 \|$ \\
Sodium nitroprusside & $329 \pm 196$ & $768 \pm 319 \ddagger$ & $421 \pm 269 \pi$
\end{tabular}

Values represent the mean \pm standard deviation.

$* p=8 \times 10^{-10}, \dagger p=0.034$, and $\neq p=0.005$ compared with normocholesterolemic $(N C)$ segments.

$\S p=0.012, \| p=0.048$, and $\| p=0.018$ compared with hypercholesterolemic control $\left(H C_{\text {control }}\right)$ segments.

Table V. Maximal contraction/relaxation force of normocholesterolemic, hypercholesterolemic control, and verapamil-treated vein grafts, expressed as percent of $\mathrm{KCl}$-induced maximal contraction

\begin{tabular}{lcll}
\hline & $N C$ & $H C_{\text {control }}$ & \multicolumn{1}{c}{$H C V_{\text {distal }}$} \\
\hline Serotonin & $270 \pm 112$ & $603 \pm 363$ & $274 \pm 152^{*}$ \\
Norepinephrine & $183 \pm 127$ & $101 \pm 62$ & $131 \pm 96$ \\
Sodium nitroprusside & $218 \pm 209$ & $192 \pm 119$ & $187 \pm 181$ \\
\hline
\end{tabular}

Values represent the mean \pm standard deviation.

${ }^{*} p=0.008$ compared with hypercholesterolemic control $\left(H C_{\text {control }}\right)$ segments.

have an additional direct mitogenic effect on SMCs, it dramatically accentuates intimal hyperplasia in rabbits, chiefly because of accumulation of lipidladen macrophages (foam cells) in the subendothelial intima. ${ }^{17,18}$ The resulting morphologic appearance of fatty plaques resembles that seen in early atherosclerotic lesions in human vein grafts. Within 4 weeks, the great increase in intimal mass allows sensitive quantitation of cellular and structural components and facilitates detection of the benefits of therapeutic interventions. The rapid course of accelerated atherosclerosis in hypercholesterolemic rabbit autologous vein grafts thus promoted the widespread use of this model for the study of possible therapeutic interventions.

Toes and coworkers ${ }^{19}$ recently reported successful inhibition of intimal and medial thickening in vein grafts by topical application of a sulfated carbohydrate polymer gel capable of absorbing heparin-binding growth factors. A potentially valuable option in peripheral vascular surgery, this semiliquid polymer relies on adjacent tissues for persistent contact with the vessel wall, making it less suitable for use in the pericardial cavity. The EVA system's features 


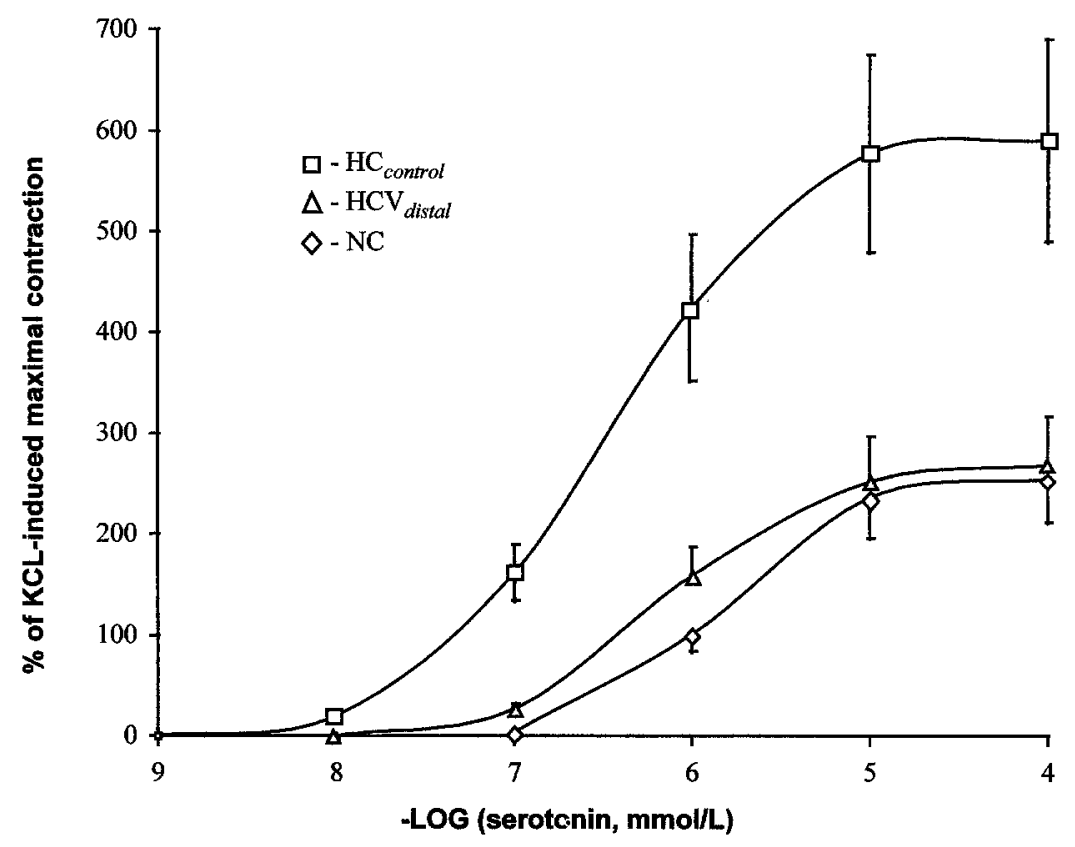

Fig. 6. Dose-response contraction curves to serotonin in distal vein graft segments. Values are percent of $\mathrm{KCl}$-induced maximal contraction and are expressed as mean \pm standard error of the mean. $H C_{\text {control }}$, Hypercholesterolemic vein graft segments, distal to empty polymers; $H V C_{\text {distal }}$, hypercholesterolemic vein graft segments distal to verapamil-EVA polymers; $N C$, normocholesterolemic graft midsegments.

make it attractive for use in coronary revascularization. This polymer, approved by the Food and Drug Administration, is both solid and highly flexible. In addition to easy application and optimal suture handling, these physical properties result in protection of the treated vessel against accidental injury. Drug release is unilaterally targeted to the adventitia by sealing one surface with blank EVA film. Determination of the drug release kinetics in physiologic buffer correlates highly with in vivo delivery and is useful to prospectively design suitable systems for any given application and animal size. By varying matrix drug loads, surface area, and thickness, the rate and duration of drug delivery can be adjusted over a wide range. ${ }^{8}$ Moreover, the study of neointimal hyperplasia mechanisms is facilitated by embedding hydrolyzable or metabolizable experimental agents in a hydrophobic polymeric controlled-release matrix. Such experimental agents (e.g., growth factors ${ }^{20,21}$ and their inhibitors ${ }^{9,10}$ ) would degrade when administered systemically, making this approach the only practical solution for examining longitudinal in vivo effects.

The calcium-channel blocker verapamil was used in the present study because of its widespread clinical availability and well-established inhibitory effects in animal models of atherosclerosis. ${ }^{13,14}$ The mechanism of antiatherogenic action of calcium channel antagonists is not entirely clear but may be due in part to direct inhibition of PDGF action. Verapamil has been shown to inhibit both independent and PDGF-induced SMC proliferation and migration in culture in a dose-dependent manner. ${ }^{11,12,14}$ In injured rabbit arteries, potent and selective inhibition of SMC proliferation occurs only when administration of these agents is begun early after injury, suggesting an interference at the $G_{0} / G_{1}$ phase of the cell cycle, possibly by prevention of modulation from the contractile to a synthetic phenotype. ${ }^{13}$ Systemic administration of verapamil $(1.25$ $\mathrm{mg} /$ day) has been shown to reduce vein graft intimal proliferation in normocholesterolemic rabbits, ${ }^{11}$ suggesting a direct inhibitory effect on intimal SMC proliferation in vivo that is independent of lipid accumulation. In the setting of hypercholesterolemia, an additional antiatherogenic action of verapamil may be due to an antioxidant effect on lowdensity lipoprotein cholesterol and to a decrease in intracellular lipid accumulation mediated by a cyclic adenosine diphosphate-dependent up-regulation of cholesteryl ester hydrolase. ${ }^{22}$

In the present study, significant reduction in vein graft intimal hyperplasia was observed in the seg- 
ments directly beneath verapamil-EVA matrices. This effect consisted mainly of inhibition of neointimal SMC proliferation, with little or no effect on lipid accumulation, as shown by the similar amount of foam cells and matrix observed in both wrapped groups. External support stents have been reported to have a beneficial effect on vein graft arterialization in pigs $^{6}$ and in hypercholesterolemic rabbits. $^{23,24}$ In particular, in a rabbit model similar to our own, intimal lipid and foam cell accumulation was significantly reduced in vein segments wrapped in polytetrafluoroethylene when compared with distal unwrapped segments. ${ }^{25}$ Unlike the matrices in the current study, however, these stents were designed to decrease wall tension by restricting vein graft distention to a predefined external diameter (4 $\mathrm{mm}$ ), often resulting in significant reduction in lumen size in addition to overall wall thickness. Moreover, Violaris and colleagues ${ }^{26}$ noted that external stenting of pig vein grafts resulted in some reduction of medial hyperplasia but also in increased intimal thickness and significant narrowing of the lumen (percent stenosis). They concluded that this technique is unlikely to be beneficial. Nevertheless, our results show reduced intimal deposition of lipid and lipid-laden cells in both verapamil-EVA and control-EVA wrapped segments when compared with the distal unwrapped segments of verapamil-treated grafts, suggesting a local beneficial effect of the polymer on intimal lipid accumulation. It seems unlikely, however, that this effect was due solely to physical external restriction, because overall graft diameter was similar under and distal to the verapamil-EVA polymers and was at least $1 \mathrm{~mm}$ smaller than the inner diameter of the applied devices. Moreover, the significant morphologic differences between vein graft segments directly under verapamil versus control matrices suggest a specific local effect of the drug, independent of any action of the polymer device.

In addition to structural remodeling, vein grafts undergo phenotypic changes that express as alterations in vasomotor function. Of these, the most prominent is a de novo expression of a serotonin type 2 (5-hydroxytryptamine 2) receptor. ${ }^{27}$ The resulting contraction response to serotonin, not observed in normal veins, has been quantitatively associated with the time-dependent development of vein graft intimal hyperplasia as expressed by the intimal index. ${ }^{27}$ Hypercholesterolemia further enhances both contraction and sensitivity of rabbit vein grafts to serotonin. ${ }^{18,28,29}$ In the present study, periadventitial administration of verapamil in hypercholesterolemic rabbits significantly reduced vein graft contraction and sensitivity to serotonin when compared with grafts treated with control empty matrices. Absolute contractile response to all agonists was also decreased. These effects were observed distal to the region of local drug delivery and were not accompanied by a reduction in measured neointimal size or net intimal SMC mass in that segment, suggesting that either a change in SMC phenotype or interference by verapamil with the vasomotor action of serotonin has occurred. The latter seems less likely, because verapamil does not affect serotonin-induced contraction in isolated arteries. ${ }^{30}$

A progressive decrease in sensitivity (but not contraction force) to norepinephrine has also been demonstrated in normocholesterolemic arterialized rabbit veins and became significant 4 weeks after implantation. ${ }^{28,31,32}$ However, hypercholesterolemia seemed to induce a supersensitivity to norepinephrine at 4 weeks in at least one report, ${ }^{28}$ a result that was not reproduced by our findings. In the present study, a slight decrease in both sensitivity and contraction to norepinephrine was observed in hypercholesterolemic control grafts, and the sensitivity (but not contraction) to norepinephrine in hypercholesterolemic vein grafts was significantly increased by verapamil treatment. Maximal relaxation to sodium nitroprusside was similar in all grafts, but the sensitivity was significantly decreased in both treated and control hypercholesterolemic grafts. The significance of observed alterations in norepinephrine and sodium nitroprusside vasoreactivity in rabbit vein grafts is not clear, and an association with intimal thickness or SMC mass was not documented. ${ }^{29}$ In addition, because of the short graft length in the rabbit model, it cannot be determined whether the effects observed distal to the region of matrix application are due to drug secretion to the bloodstream or to local diffusion within the vessel wall.

The ability of a periadventitial drug delivery system to locally generate specific inhibition of neointimal SMC proliferation presents a potentially convenient alternative to systemic drug administration for both therapeutic and research purposes. This method seems readily applicable in coronary revascularization operations. Further evaluation of delivery systems with various agents and doses may optimize this approach for the purpose of clinical use.

We gratefully acknowledge the technical assistance of Jannis Cuevas. 


\section{REFERENCES}

1. Grondin CM, Campeau L, Thornton JC, et al. Coronary artery bypass grafting with saphenous vein. Circulation 1989; 79(6 Pt 2):124-9.

2. Zwolak RM, Adams MC, Clowes AW. Kinetics of vein graft hyperplasia: association with tangential stress. J Vasc Surg 1987;5:126-36.

3. Cox JL, Chiasson DA, Gotlieb AI. Stranger in a strange land: the pathogenesis of saphenous vein graft stenosis with emphasis on structural and functional differences between veins and arteries. Prog Cardiovasc Dis 1991;34:45-68.

4. Herrman JP, Hermans WR, Vos J, Serruys PW. Pharmacological approaches to the prevention of restenosis following angioplasty. The search for the Holy Grail? (Part I). Drugs 1993;46:18-52.

5. Golomb G, Moscovitz D, Fishbein I, Mishaly D. Perivascular polymeric controlled-release therapy. In: Wise DL, editor. Encyclopedic handbook of biomaterials and bioengineering. New York: Marcel Dekker; 1995. p. 1043-55.

6. Angelini GD, Izzat MB, Bryan AJ, Newby AC. External stenting reduces early medial and neointimal thickening in a pig model of arteriovenous bypass grafting. J Thorac Cardiovasc Surg 1996;112:79-84.

7. Edelman ER, Adams DH, Karnovsky MJ. Effect of controlled adventitial heparin delivery on smooth muscle cell proliferation following endothelial injury. Proc Natl Acad Sci U S A 1990;87:3773-7.

8. Golomb G, Fishbein I, Banai S, et al. Controlled delivery of a tyrphostin inhibits intimal hyperplasia in a rat carotid artery model. Atherosclerosis. 1996;125:171-82.

9. Simons M, Edelman ER, DeKeyser JL, et al. Antisense c-myb oligonucleotides inhibit intimal arterial smooth muscle cell accumulation in vivo. Nature 1992;359:67-70.

10. Simons M, Edelman ER, Rosenberg RD. Antisense proliferating cell nuclear antigen oligonucleotides inhibit intimal hyperplasia in a rat carotid artery injury model. J Clin Invest 1994;93:2351-6.

11. el-Sanadiki MN, Cross KS, Murray JJ, et al. Reduction of intimal hyperplasia and enhanced reactivity of experimental vein bypass grafts with verapamil treatment [see comments]. Ann Surg 1990;212:87-96.

12. Yang $Z$, Noll G, Luscher TF. Calcium antagonists differently inhibit proliferation of human coronary smooth muscle cells in response to pulsatile stretch and platelet-derived growth factor. Circulation 1993;88:832-6.

13. Jackson CL, Bush RC, Bowyer DE. Mechanism of antiatherogenic action of calcium antagonists. Atherosclerosis 1989;80:17-26.

14. Waters D, Lesperance J. Calcium channel blockers and coronary atherosclerosis: from the rabbit to the real world. Am Heart J 1994;128(6 Pt 2):1309-16.

15. Golomb G, Langer R, Schoen FJ, et al. Controlled release of diphosphonate to inhibit bioprosthetic heart valve calcification: dose-response and mechanistic studies. J Controlled Rel 1986;4:181-94.

16. Ip JH, Fuster V, Badimon L, et al. Syndromes of accelerated atherosclerosis: role of vascular injury and smooth muscle cell proliferation. J Am Coll Cardiol 1990;15:1667-87.

17. Zwolak RM, Kirkman TR, Clowes AW. Atherosclerosis in rabbit vein grafts. Arteriosclerosis 1989;9:374-9.

18. Davies MG, Kim JH, Barber L, et al. Systemic hypertension and hypercholesterolemia in vein grafts: effects on the function and morphology of experimental vein grafts. J Surg Res 1994;57:106-21.

19. Toes GJ, Barnathan ES, Liu $\mathbf{H}$, et al. Inhibition of vein graft intimal and medial thickening by periadventitial application of a sulfated carbohydrate polymer. J Vasc Surg 1996;23:650-6.

20. Jawien A, Bowen-Pope DF, Lindner V, et al. Platelet-derived growth factor promotes smooth muscle migration and intimal thickening in a rat model of balloon angioplasty. J Clin Invest 1992;89:507-11.

21. Edelman ER, Nugent A, Smith LT, Karnovsky MJ. Basic fibroblast growth factor enhances the coupling of intimal hyperplasia and proliferation of vasa vasorum in injured rat arteries. J Clin Invest 1992;89:465-73.

22. Henry PD. Antiperoxidative actions of calcium antagonists and atherogenesis. J Cardiovasc Pharmacol 1991;18(Suppl 1):S6-10.

23. Zweep HP, Satoh S, van der Lei B, et al. Degradation of a supporting prosthesis can optimize arterialization of autologous veins. Ann Thorac Surg 1993;56:1117-22.

24. Zweep HP, Satoh S, van der Lei B, et al. Autologous vein supported with a biodegradable prosthesis for arterial grafting. Ann Thorac Surg 1993;55:427-33.

25. Batellier J, Wassef M, Merval R, et al. Protection from atherosclerosis in vein grafts by a rigid external support. Arteriosclerosis Thromb 1993;13:379-84.

26. Violaris AG, Newby AC, Angelini GD. Effects of external stenting on wall thickening in arteriovenous bypass grafts. Ann Thorac Surg 1993;55:667-71.

27. Radic ZS, O'Donohoe MK, Schwartz LB, et al. Alterations in serotonergic receptor expression in experimental vein grafts. J Vasc Surg 1991;14:40-7.

28. Klyachkin ML, Davies MG, Svendsen E, et al. Hypercholesterolemia and experimental vein grafts: accelerated development of intimal hyperplasia and an increase in abnormal vasomotor function. J Surg Res 1993;54:451-68.

29. Klyachkin ML, Davies MG, Kim JH, et al. Postoperative reduction of high serum cholesterol concentrations and experimental vein bypass grafts: effect on the development of intimal hyperplasia and abnormal vasomotor function. J Thorac Cardiovase Surg 1994;108:556-66.

30. Villalon CM, Ramirez-San Juan E, Castillo C, et al. Pharmacological profile of the receptors that mediate external carotid vasoconstriction by $5-\mathrm{HT}$ in vagosympathectomized dogs. Br J Pharmacol 1995;116:2778-84.

31. Makhoul RG, Davis WS, Mikat EM, et al. Decreased sensitivity to norepinephrine in vein bypass grafts. Curr Surg 1987;44:46-8.

32. Makhoul RG, Davis WS, Mikat EM, et al. Responsiveness of vein bypass grafts to stimulation with norepinephrine and 5-hydroxytryptamine. J Vasc Surg 1987;6:32-8. 